\title{
Comunicação
}

[Communication]

\section{Avaliação da técnica de arteriografia renal guiada por fluoroscopia em suínos}

\author{
[Description of the fluoroscopy-guided renal arteriography in swine]
}

\author{
C.A.P. Sarmento ${ }^{1}$, E. Branco ${ }^{2 *}$, E.T. Fioretto ${ }^{3}$, G.J. Ferreira ${ }^{4}$, G.B. Gregores ${ }^{1}$, \\ A.R. Lima ${ }^{2}$, W.F. Silva ${ }^{1}$, R. Cabral ${ }^{5}$, M.A. Miglino ${ }^{1}$ \\ ${ }^{1}$ Faculdade de Medicina Veterinária e Zootecnia - USP- São Paulo, SP \\ ${ }^{2}$ Instituto de Saúde e Produção Animal - FMV-UFRA - Belém, PA \\ ${ }^{3}$ Centro de Ciências Biológicas e da Saúde - UFSE - Aracaju, SE \\ ${ }^{4}$ Universidade Federal do Piauí - Bom Jesus, PI \\ ${ }^{5}$ Centro de Ciências Agrárias - FMV-UFPI - Teresina, PI
}

A importância do diagnóstico precoce dos pacientes com suspeita de alteração renovascular advém do risco de progressão para a insuficiência renal devido à isquemia nefropática causada por lesões limitantes de fluxo na artéria renal. Nos casos de nefropatia vascular humana, o diagnóstico pode ser feito por diferentes exames complementares caros e complexos como: determinação da renina seletiva na veia renal, estudo funcional com radioisótopos, urografia excretora, determinação de renina periférica estimulada com captopril, angiorressonância magnética e ultrassom vascular com Doppler colorido (Engelhorn et al., 2004), porém estes exames não fazem parte da rotina da medicina veterinária.

A arteriografia, do ponto de vista anatômico, apresenta-se como o método mais preciso para o diagnóstico de estenose de artéria renal, sendo complementar de outros exames que forneçam informações hemodinâmicas.

Seldinhger (1953) descreveu uma técnica realizada por via percutânea, que consistiu na punção de um vaso, mediante uma agulha inserida através da pele, por onde se introduziu um fio-guia, que serviu de sustentação para a introdução de um cateter, o que permitiu a cateterização seletiva de praticamente todos os principais territórios vasculares do organismo, facilitou a avaliação e interpretação das angiografias, e permitiu dissociar as estruturas vasculares mais complexas, com menor necessidade de volume de contraste e maior concentração do contraste nas artérias-alvo. Essa descoberta fez com que o exame radiográfico simples, para avaliações vasculares, fosse colocado de lado, pois esse tipo de análise não era capaz de proporcionar o diagnóstico de algumas lesões, visto que o tecido mole e os vasos sanguíneos apresentam densidade radiográfica muito semelhante (Burk e Acherman 1996).

Assim, o presente estudo teve o objetivo de avaliar a técnica da arteriografia renal guiada por fluoroscopia, uma vez que o suíno é constantemente estudado como modelo experimental de diversas patologias incluindo as de cunho renal. Todo procedimento esteve de acordo com as normas do Comitê de Bioética da Faculdade de Medicina Veterinária da Universidade de São Paulo, sob o processo $n^{\circ}$ 750/2005.

Foram utilizados seis suínos, adultos, hígidos, fêmeas, com aproximadamente $20 \mathrm{Kg}$, obtidos de granjas na região da cidade de São Paulo.

Recebido em 29 de outubro de 2010

Aceito em 22 de fevereiro de 2011

* Autor para correspondência (corresponding author)

E-mail: erika.branco@ufra.edu.br 
A via de acesso à artéria renal deu-se pela cateterização da artéria femoral esquerda. Para a realização dos procedimentos de cateterização da artéria femoral, os animais foram submetidos à aplicação de pré-anestésico, por meio da associação de cloridrato de ketamina (3mg/kg) e midazolam $(0,5 \mathrm{mg} / \mathrm{kg})$, intramuscular, e à indução feita mediante uso de propofol intravenoso $(5 \mathrm{mg} / \mathrm{kg})$. A manutenção foi feita usando-se isofluorane e ventilação controlada a pressão $(8 \mathrm{~mL} / \mathrm{kg})$ com $\mathrm{FR}=15$ e $\mathrm{PEEP}=5 \mathrm{~cm}$ $\mathrm{H}_{2} \mathrm{O}$ (pressão respiratória final das vias aéreas).

Com o animal em plano anestésico, realizaramse a dissecação e a canulação da artéria femoral esquerda, utilizando-se de uma Fita Cardíaca ${ }^{\circledR}$ (Ethicon - Johnson \& Johnson) para ancoragem. Um cateter guia flexível, através de uma bainha, foi guiado à artéria renal esquerda, seguindo o seguinte trajeto: artéria femoral esquerda, artéria ilíaca esquerda, aorta abdominal, artéria renal esquerda, sendo esse o trajeto padrão para todos os animais. O direcionamento, a localização do catéter e a visualização da artéria renal esquerda foram promovidos pela injeção de solução de contraste iodado (Telebrix Coronar ${ }^{\circledR}$ - Guerbet), e percepção da imagem por meio de fluoroscopia (Fluoroscópio Philips BV Pulsera). Finalizada a arteriografia e a aquisição das imagens, procedeu-se à ligadura da artéria femoral seguindo-se a sutura da região.

A arteriografia renal mostrou-se um exame passível de ser realizado, apesar de ser algo bastante delicado, porém absolutamente indicado na avaliação de processos patológicos que envolvam a vascularização renal, principalmente na espécie suína, bastante utilizada como modelo experimental de diversas desordens, incluindo as nefrológicas.

A infusão do meio de contraste positivo preencheu as artérias setoriais cranial e caudal e proporcionou excelente qualidade da imagem radiográfica dos ramos segmentares que vascularizam territórios independentes em cada setor renal, caracterizando os segmentos renais (Figure 1).

O objetivo principal dos exames complementares nas investigações de portadores de lesão renovascular é identificar as lesões anatômicas nas artérias renais e seus ramos, bem como determinar quais lesões serão fisiologicamente significativas, isto é, responsáveis pela ativação do sistema renina-angiotensina (Haimovici e Zinicola, 1962).

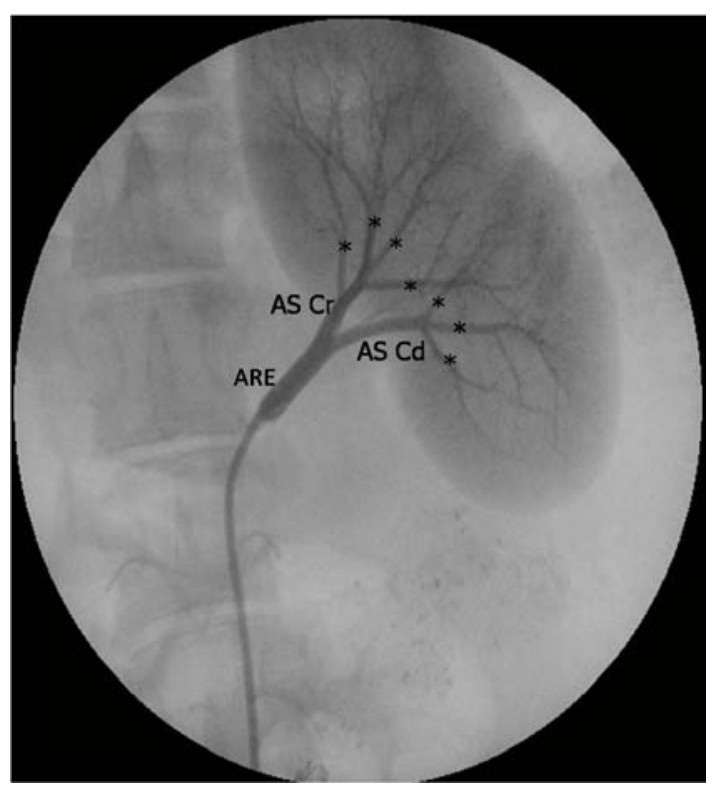

Figura 1. Imagem angiográfica do rim esquerdo evidenciando artéria renal esquerda (ARE), artéria setorial cranial (ASCr), artéria setorial caudal (ASCd) e os ramos segmentares $(*)$.

Apesar de simples, passível de ser reproduzida e considerada o exame de escolha para o diagnóstico anatômico das lesões estenosantes ou obstrutivas das artérias renais, a arteriografia não fornece informações hemodinâmicas (Hessel et al., 1981; Waugh e Sacharias, 1982; Egglin et al., 1995; Jaarsveld et al., 1999), logo deve ser considerada como exame complementar. De acordo com Engelhorn et al. (2004), sempre que for possível utilizar métodos menos invasivos, tais como a ultrassonografia com Doppler colorido antes da avaliação angiográfica, a arteriografia é indicada.

O meio de contraste positivo iodado possui elevada radiodensidade, que é determinada pela quantidade relativa de iodo presente em cada molécula. A viscosidade, que se relaciona com o tamanho da partícula, também é determinante para a facilidade e rapidez da administração do contraste e, se for elevada, pode resultar em complicações, principalmente quando se usam cateteres de pequeno calibre (Dennis, 2009). O contraste usado neste estudo (Telebrix Coronar ${ }^{\circledR}$ - Guerbet), apesar de sua viscosidade, não 
interferiu na administração intravenosa, diferente do observado por Burk e Ackerman (1996).

A aplicação do exame de arteriografia renal guiada por fluoroscopia ainda não é uma técnica frequente no auxílio de diagnósticos na clínica médica em medicina veterinária, tal como se observa na medicina humana, desse modo é mais utilizada no âmbito experimental. Esse fato devese, na prática, ao alto custo do equipamento, à improbabilidade de retorno do investimento e à falta de domínio da técnica por parte dos profissionais da medicina veterinária (Machado, 1992), porém a arteriografia é considerada padrão ouro para o diagnóstico de estenose renal. A arteriografia convencional é a melhor técnica recomendada quando não há possibilidade de emprego da angiografia digital ou quando esta não fornece imagens adequadas, o que ocorre em 13\% dos casos (Ram et al., 1995), pois permite a identificação dos segmentos independentes, irrigados por meio de artérias segmentares individualizadas, o que torna possível sua remoção cirúrgica (DiDio e Motta, 1985; DiDio, 1999).

Com base nos resultados obtidos, concluiu-se que a técnica de arteriografia renal em suínos por método de fluoroscopia é viável e pode contribuir com investigações de patologias vasculares que acometem os rins. Quando induzidas experimentalmente nesta espécie, é bastante utilizada como modelo de diversas doenças, porém esta técnica requer habilidade por parte dos profissionais no que tange ao processo de cateterização.

Palavras-chave: angiografia renal, fluoroscopia, suínos

\begin{abstract}
The fluoroscopy-guided renal arteriography was evaluated in order to be used as an auxiliary method in investigations and as a way to experimentally induce kidney diseases in swine. The technique was effective to obtain sharp images as well as to determine the area of renal irrigation. Despite its easiness, trained professionals are required to perform it.
\end{abstract}

Keywords: renal angiography, fluoroscopy, swines

\section{AGRADECIMENTOS}

À Fundação de Amparo à Pesquisa do Estado de São Paulo, FAPESP, e ao Instituto do Coração INCOR de São Paulo, pelo financiamento e oportunidade oferecida a um projeto que subsidia pesquisas básicas, e que poderá ser empregado como forma investigativa em outros estudos.

\section{REFERÊNCIAS BIBLIOGRÁFICAS}

BURK, R.L.; ACKERMAN, N. Small animal radiology and ultrasonography: A diagnostic atlas and text. 2.ed. Philadelphia: W.B. Saunders, 1996. 740p.

DENNIS, R. Use of contrast media in veterinary radiology. Disponível em:

$<$ http://www.vin.com/proceedings/Proceedings.p lx?CID=WSAVA2002\&PID=2606 $>$. Acessado em: 15 out. 2009.
DIDIO, L.J.A. Tratado de anatomia aplicada. São Paulo: Pólus Comunicação Integrada, 1999. 948p.

DIDIO, L.J.A.; MOTTA, P. Segments of the kidney the anatomical basis for nephodsegmentectomy. In: DIDIO, L.J.A.; MOTTA, P.M. (Eds). Basic clinical and surgical nephrology. Boston: Martinus Nijhoff, 1985. p.112

EGGLIN, T.O.; MOORE, P.V.; FEINSTEIN, A.R. et al. Complications of peripheral arteriography: A new system to identify patients at increased risk. J. Vasc. Surg., v.22, p.787-794, 1995.

ENGELHORN, C.A.; ENGELHORN, A.L.; PULLIG, R. O papel da ultrassonografia vascular com doppler colorido na avaliação da hipertensão renovascular. acurácia da técnica direta de avaliação das artérias renais. Arq. Bras. Cardiol., v.82, p.473-476, 2004. 
HAIMOVICI, H., ZINICOLA, N. Experimental renal artery stenosis: diagnostic significance of arterial hemodinamics. J. Cardiovasc. Surg., v.3, p.259-262, 1962.

HESSEL, S.J.; ADAMS, D.F.; ABRAMS, H.L. Complications of angiography. Radiology, v.138, p.273-281, 1981.

JAARSVELD, B.C.; PIERTERMAN, H.; DIJIK, L.C. et al. Interobserver variability in the angiographic assessment of renal artery stenosis. J. Hyperten., v.17, p.1731-1736, 1999.
MACHADO, C.C. Criterios cubanos para el diagnostico de la muerte encefalica. Ciudad de La Habana: Editorial Ciencias Médicas, 1992.

RAM, C.V.S.; CLAGETT, G.P.; DARFORD, L.R. Renovascular hypertension. Semin. Nephrol. J., v.5, p.152-174, 1995.

SELDINGER, S. Catheter replacement of the neelie in percuta-neous arteriography. Acta Radiol., v.39, p.368, 1953.

WAUGH, J.R.; SACHARIAS, N. Arteriography complications in DAS era. Radiology, v.182, p.243-246, 1992. 